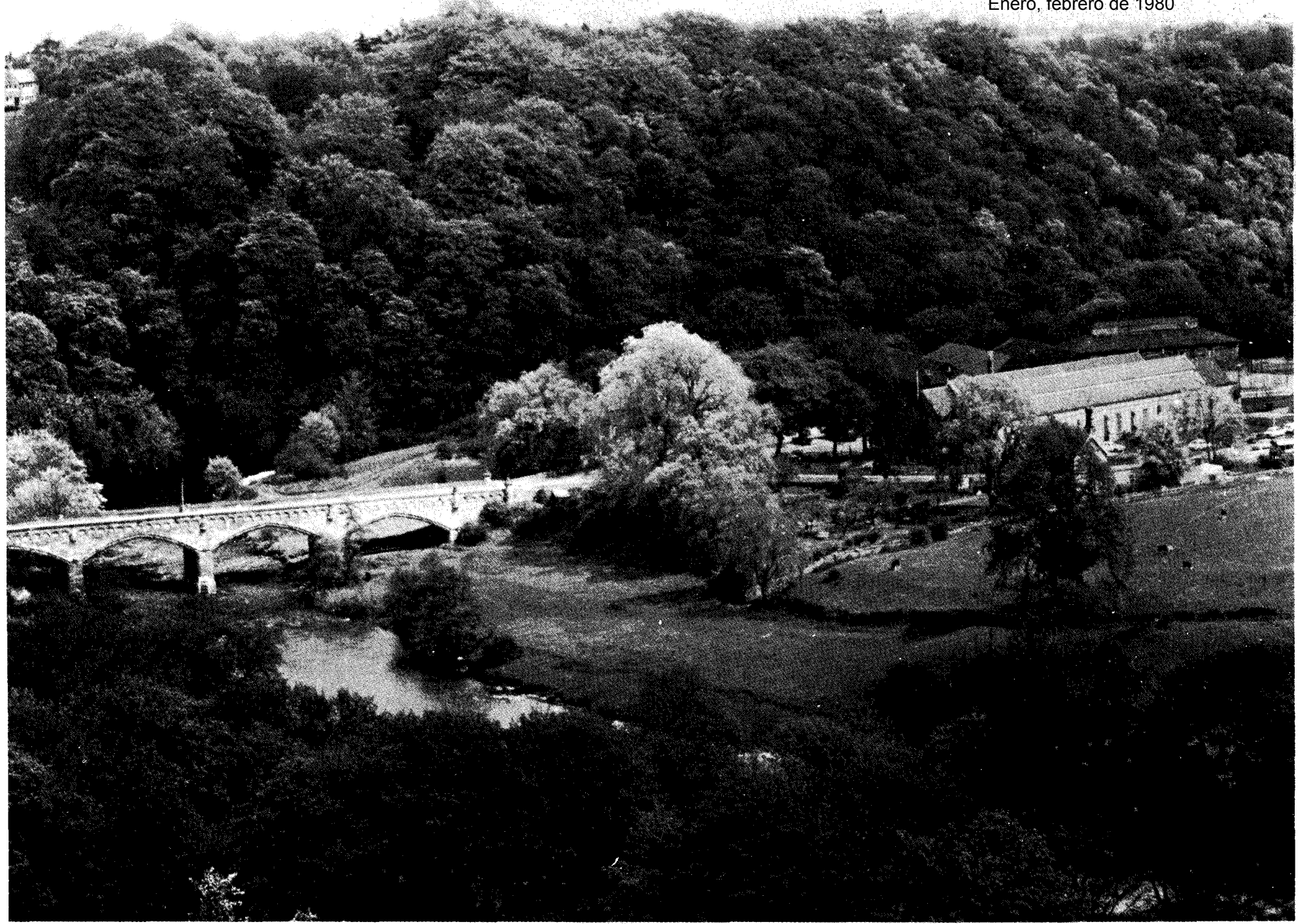

\title{
Piscina cubierta integrada en un conjunto de edificios Victorianos Gran Bretaña
}

\section{Sinopsis}

$151-39$

Se presenta un edificio moderno, dedicado a piscina cubierta, muy bien encajada con la inmediata estación victoriana de mediados del siglo XIX.

Consta de dos plantas: inferior con piscina de no nadadores, de $12 \times 7 \mathrm{~m}$, vestuarios $\mathrm{y}$ piscina de competición, de $25 \times 12,50 \mathrm{~m}$; la planta superior alberga las taquiIllas, dirección, administración, cafetería, etcétera con una terraza desde la que se disfrutan vistas magníficas.

La estructura es metálica y se ha procurado quitarle monotonía, a fin de conjugar el modernismo $y$ funcionalidad, con el entorno no paisajístico y arquitectónico. 
A unos $350 \mathrm{~km}$ al norte-noroeste de Londres, a mitad del camino entre Leeds o York (en la zona industrial del centro de Inglaterra) y Newcastle (en la frontera inglesa-escocesa) se encuentra la pequeña ciudad de Darlington. Más o menos $20 \mathrm{~km}$ al suroeste de ésta se ubica la localidad de RICHMOND, en la parte norte del condado de Yorkshire, en uno de los paisajes más bellos de Inglaterra, en la pintoresca cuenca del río Swale. El castillo histórico, construido en 1071 por Alain le Rouge, después de que Guillermo el Descubridor le concediera la tierra en feudo, es el centro de la ciudad. A la salida de la misma se encuentra una estación neogótica, construida por George T. Andrews en 1848 y actualmente un ejemplo notable de la arquitectura ferroviaria de aquellos tiempos, que está considerada como monumento. Esta estación de la línea de Darlington clausurada en 1969, fue adquirida por el municipio en 1972, restaurada y dedicada desde entonces a constituir el conjunto de un centro deportivo regional. Estaban previstas pistas de tenis, squash y deportivas, además una piscina - proyectada en detalle en 1976 como primera fase de construcción de un gran centro deportivo cubierto- y parque de descanso a lo largo del río Swale.

La planta inferior de la piscina alberga en la parte inclinada los vestuarios. Sigue la piscina para los no nadadores de $12 \times 7 \mathrm{~m}$, a la cual se accede a través de varios escalones de poca altura y muy anchos, que permiten que incluso los niños pequeños puedan familiarizarse poco a poco con el aqua. A continuación se encuentra la piscina de cinco pistas de $12,50 \times 25 \mathrm{~m}$, y profundidad de $1,70 \mathrm{~m}$ hasta $0,90 \mathrm{~m}$, renunciándose conscientemente a los trampolines, ya que la piscina sirve más a la natación de descanso que a la natación competitiva.

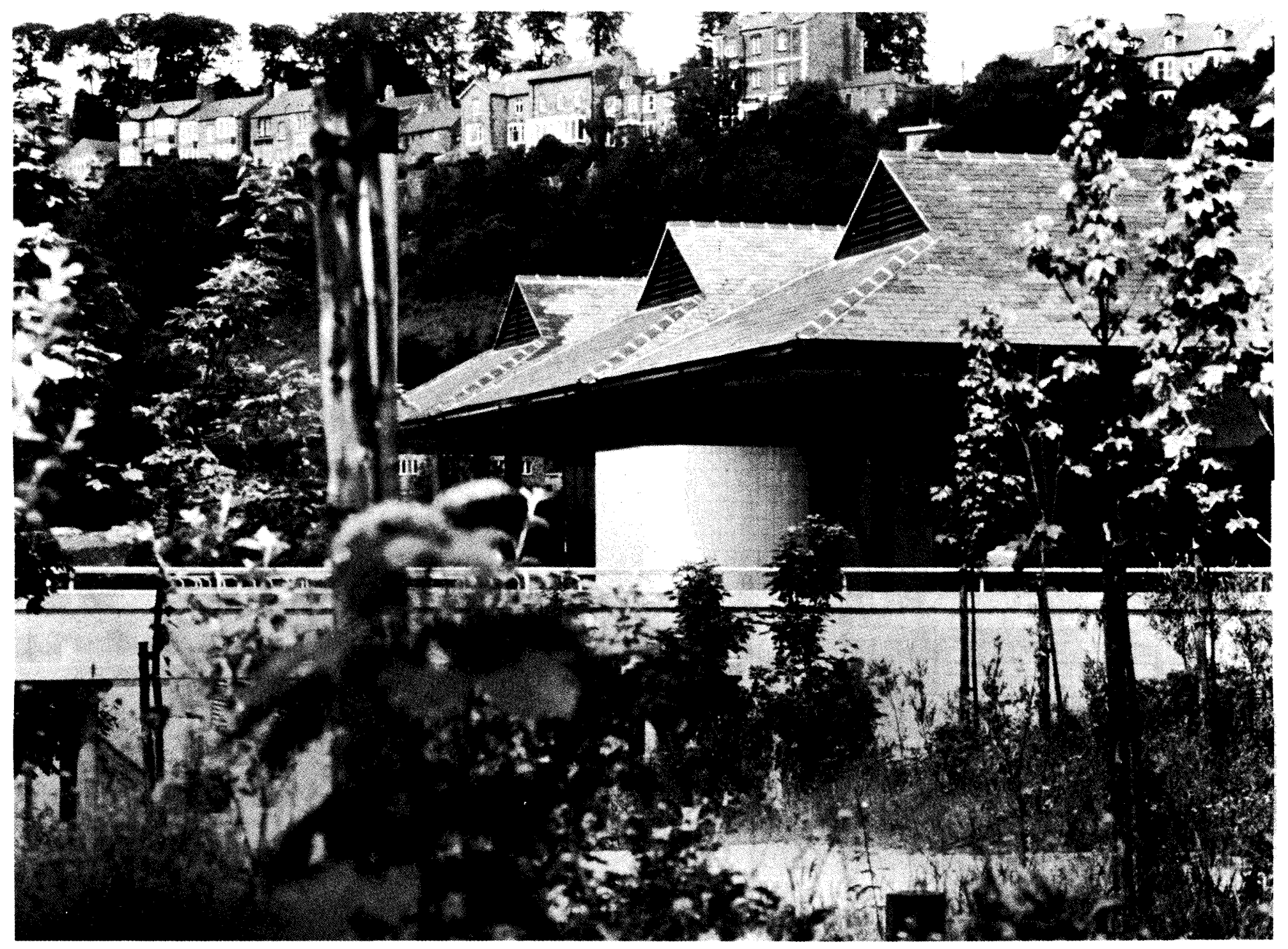



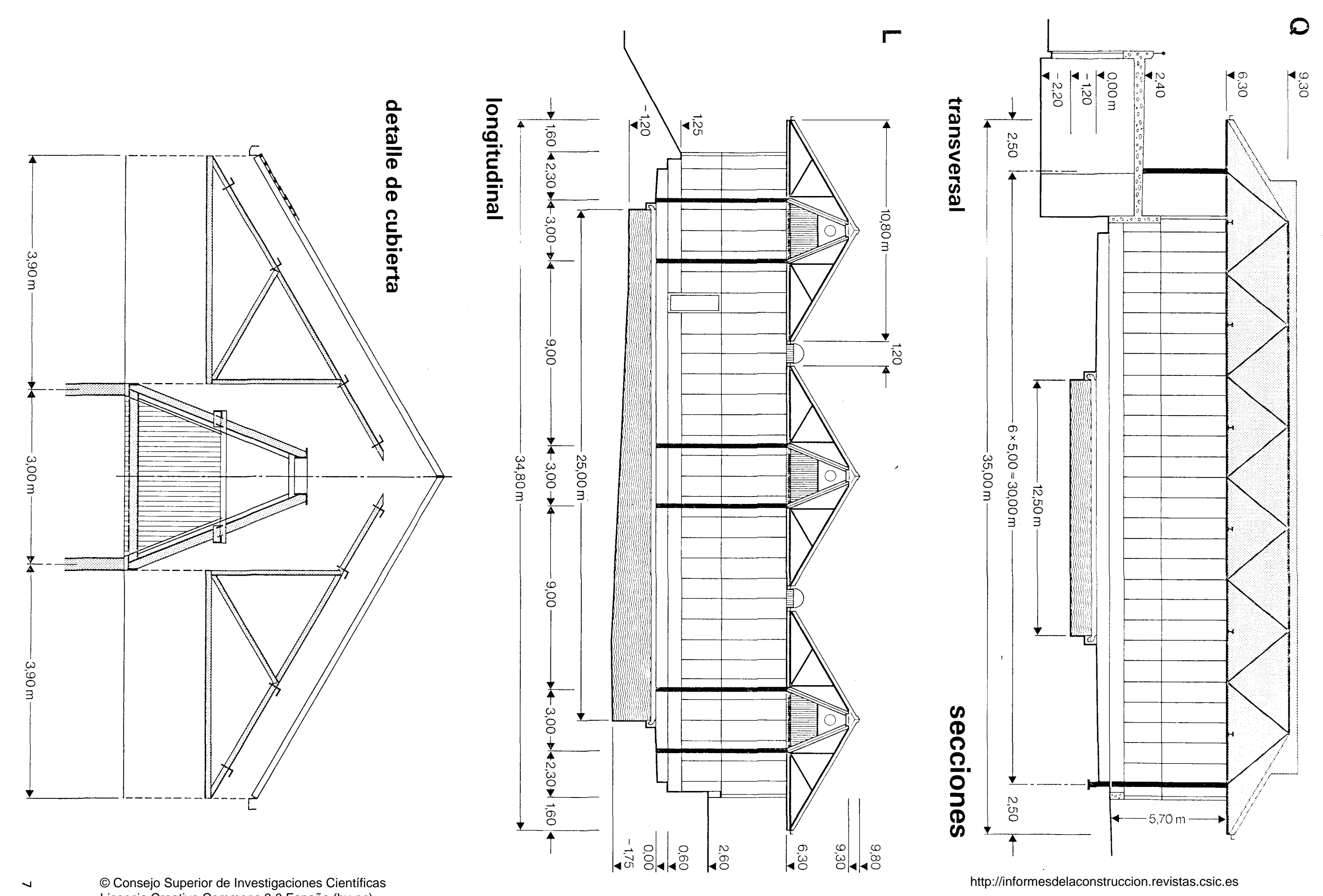

(C) Consejo Superior de Investigaciones Científicas Licencia Creative Commons 3.0 España (by-nc) 
En la planta superior de la piscina se encuentra la entrada principal, la sala anterior con las taquillas, salas de dirección y personal, cafetería y bar de bebidas no alcohólicas; este último sirve por una parte como tribuna y ofrece una buena vista de la piscina. Por otra parte se ve desde el mismo la estación histórica y una magnífica terraza cubierta. La piscina cubierta se adapta de tal forma al terreno inclinado, que su planta superior pudo unirse directamente con la estación por medio de una pasarela de peatones por encima de la carretera de acceso, una separación limpia de dos corrientes de tráfico.

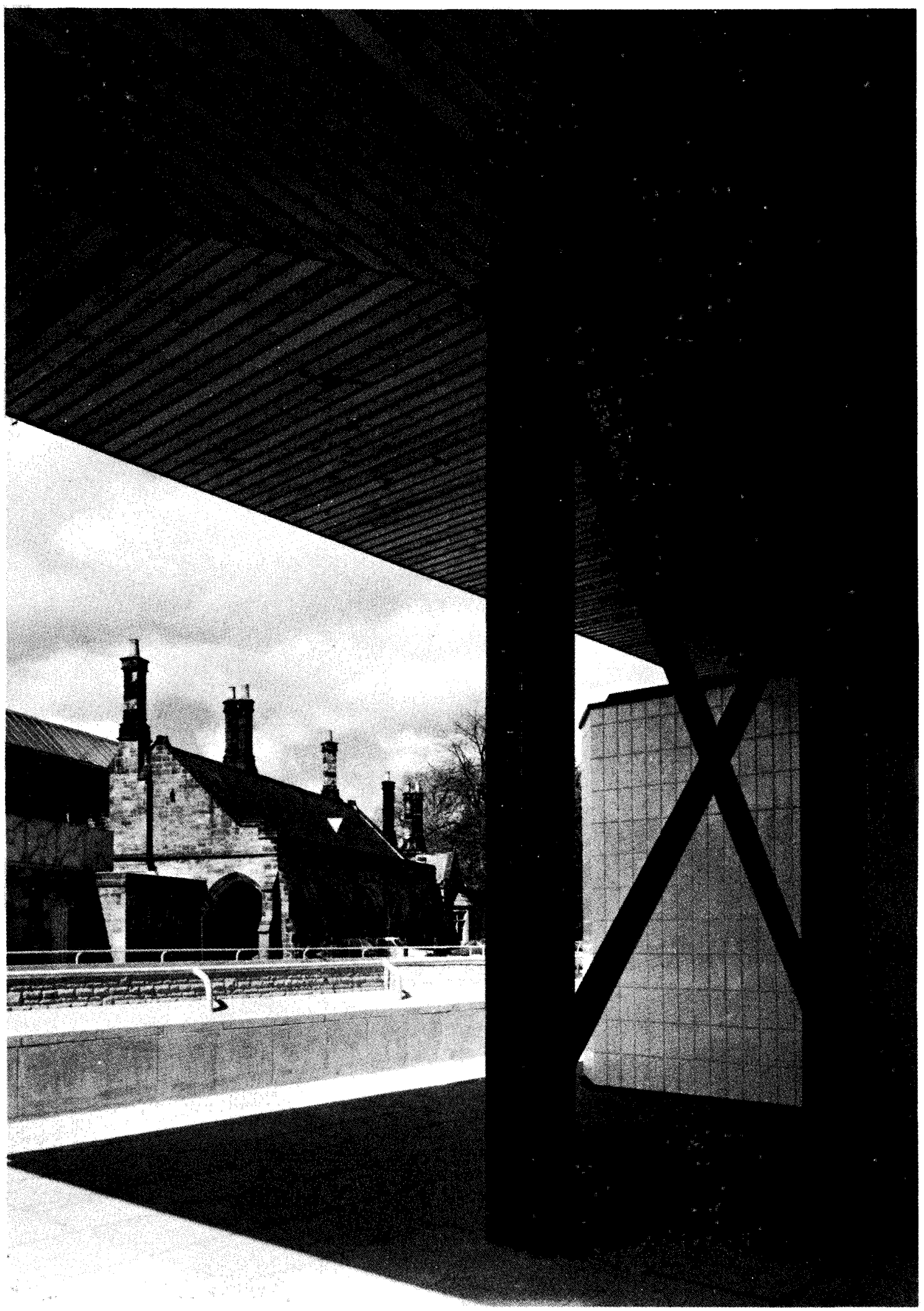



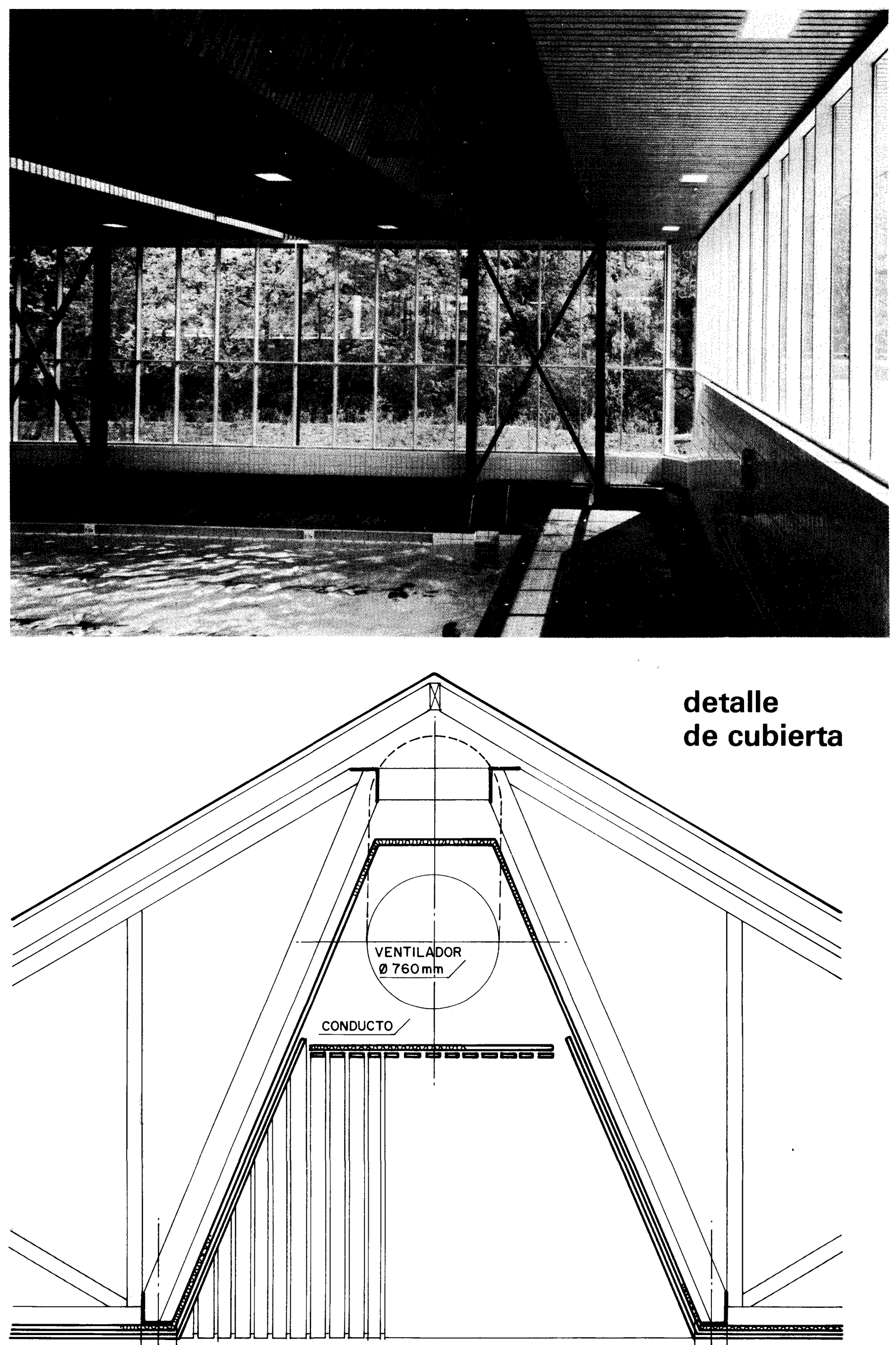

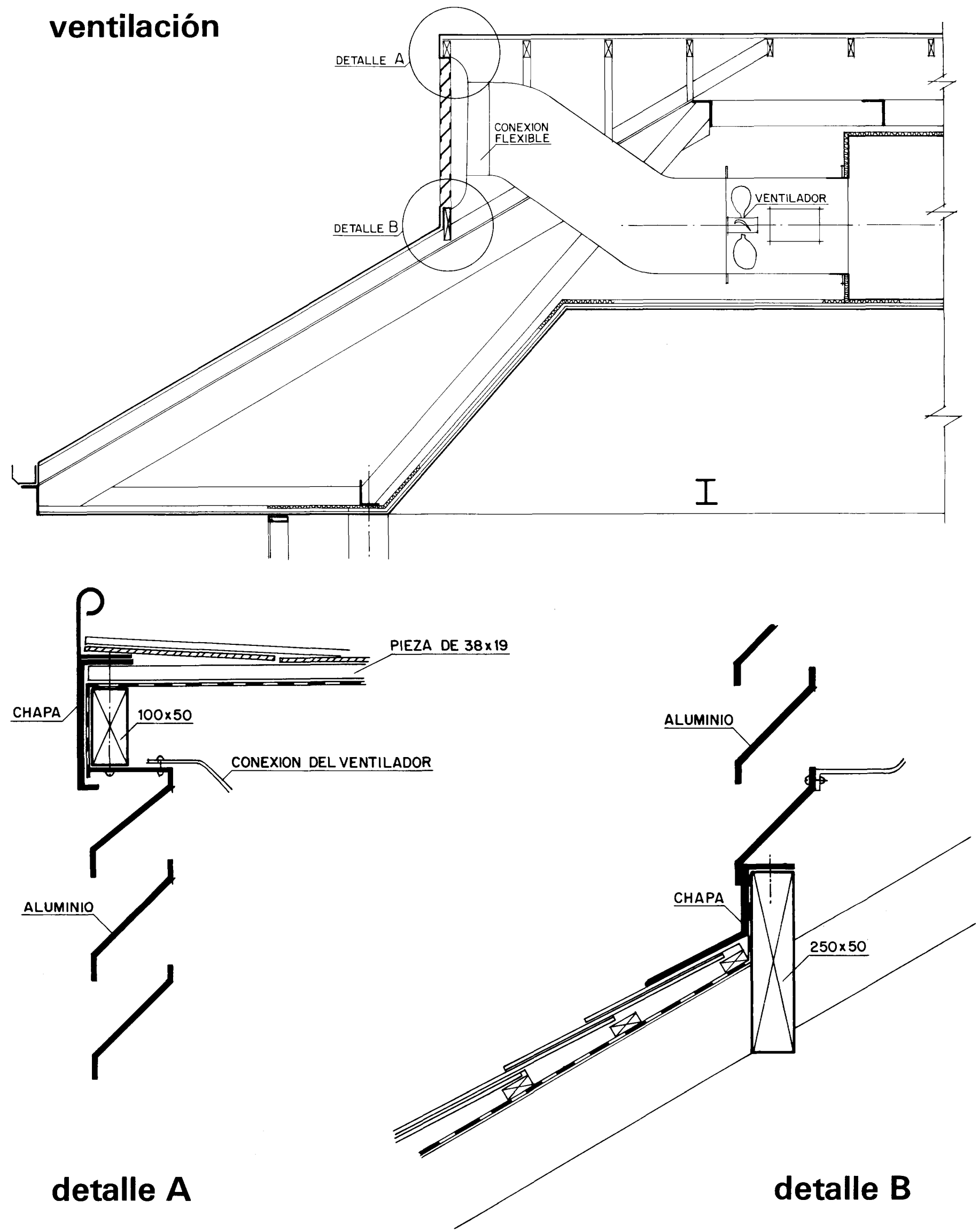

El cuerpo de construcción de la piscina se distingue agradablemente de la monótona forma de caja de las piscinas cubiertas usuales, porque el frente se ha dividido y el techo queda con tres frontones separados, que recuerdan a una pagoda china. Los tres frontones de la cubierta están situados en sentido transversal, con respecto al lado más largo de la piscina. 
La techumbre de acero se compone de un frontón, vigas de celosía transversales con seis cerchas triangulares longitudinales en los pares de apoyos de ambos extremos. Cada par de vigas de celosía consta respectivamente de dos vigas de celosía en $\mathrm{W}$ inclinadas ligeramente una hacia la otra, con una envergadura de $30 \mathrm{~m}$ y una longitud total de $35 \mathrm{~m}$, incluyendo los voladizos, a ambos lados, de 2,5 m, con tornapuntas de $50^{\circ}$, una distancia entre pilares de $3 \mathrm{~m}$ y una altura de $3 \mathrm{~m}$. En el cordón superior las dos vigas de celosía se unen por medio de perfiles $L$ de $0,6 \mathrm{~m}$ de longitud y en el cordón inferior por medio de perfiles $\mathrm{C}$ de $3 \mathrm{~m}$ de longitud. Todos ellos forman, con los pilares y las cerchas triangulares, un plano perpendicular que resaltan a la vista desde abajo de la nave. Las seis «cerchas triangulares» se han dispuesto en dos mitades simétricas ortogonalmente sobre las vigas de celosía, teniendo cada frontón una envergadura de casi $11 \mathrm{~m}$ y resultando una longitud total cubierta de $33 \mathrm{~m}$. Los seis pares de apoyos se unen respectivamente en un bloque rígido por medio de una unión en cruz de San Andrés. No impiden la vista desde la piscina hacia el paisaje. Estáticamente el par de vigas de celosía, actúa como viga en cajón solicitada por torsión, debido a las mitades salientes de las cerchas triangulares, por lo que ha de construirse fuertemente a fin de limitar la torsión y como consecuencia la inclinación de los voladizos. Para estabilizar el tejado se disponen en el plano del cordón inferior de las vigas de celosía y cerchas triangulares unas uniones en $W$ en el borde de la cubierta así como en el centro de la nave, en sentido transversal. El espacio intermedio se aprovecha muy bien para instalar los canales de aire. La salida del aire se reconoce por las rejillas de los extremos.

A fin de incorporar la piscina en el paisaje y adaptarla a la estación histórica, se cubrieron sus tres faldones con pizarra. Su zócalo es de piedra arenisca, nuevamente labrada, procedente de unos antiguos depósitos de locomotoras, que ya no podían ser restaurados.

Dipl.-Ing. K. Idelberger

\section{PROYECTO Y EJECUCION}

Constructor. District Council of Richmondshire.

Arquitectos: Napper Errington Collerton Partnership, Newcastle upon Tyne.

Ingenieros: Ove Arup \& Partners, Londres.

Estructura: T. J. Newton.

Fotos: Henk Snoek Photo \& Ass., Hampstead.

Résumé

PISCINE COUVERTE INTEGREE DANS UN ENSEMBLE DE BATIMENTS (STATION) VICTORIENS -GRANDEBRETAGNE

N. E. Collerton Part., architectes Ove Arup \& Part., ingénieurs

Il s'agit là đ'un bâtiment moderne, destiné à une piscine couverte, qui s' harmonise très bien avec la station victorienne immédiate du milieu du XIXe siècle.

II comprend deux niveaux: le niveau inférieur est occupé par la piscine de non nageurs, de $12 \times$ $7 \mathrm{~m}$, les vestiaires et la piscine de compétition de $25 \times 12,50 \mathrm{~m}$; le niveau supérieur abrite les guichets, les bureaux de direction et d administration, la cafeteria, etc. avec une terrasse d'où I'on a des vues magnifiques.

La structure, métallique, a été conçue de telle façon que la monotonie a été évitée afin de conjuguer le modernisme et le caractère fonctionnel avec l'architecture et le paysage environtionnel
nants.

\section{Summary}

AN IN-DOOR SWIMMING POOL IN THE MIDDLE OF A GROUP OF VICTORIAN BUILDINGS (A RAILWAY STATION UNITED KINGDOM

N. E. Collerton Part., architects

Ove Arup \& Part., engineers

This modern building, housing an in-door swimming pool, blends-in beautifully with the adjacent mid-nineteen Century Victorian railway station.

The building has two floors; the ground floor with a $12 \times 7 \mathrm{~m}$ wading pool, a $25 \times 12.5 \mathrm{~m}$ competition pool and dressing rooms, while the upper floor houses the lockers, management office, cafeteria, etc. and a terrace enjoying magnificent views.

The steel structure was designed avoiding the sternness of moderm functionalism in an attempt to make it compatible with architectural environment.

\section{Zusammenfassung}

UEBERDACHTES SCHWIMMBAD INNERHALB DES VIKTORIANISCHEN GEBAEUDEKOMPLEXES (BAHNHOF) GROSS BRETANIEN

\section{Architekten: N. E. Collerton Part.} Ingenieurs: Ove Arup \& Part.

Es wird ein modernes Gebäude, ein überdachtes Schwimmbad, vorgestellt, das in ausgezeichneter Weise der benachbarten viktorianischen Station, welche Mitte des XIX Jahrhunderts errichtet wurde, angepasst ist.

Dieses Gebäude besteht aus zwei Stockwerken: im unteren befinden sich das NichtschwimmerBecken mit Abmessungen von $12 \times 7 \mathrm{~m}$, Umkleideräume, sowie das Becken für Wett ampfe von $25 \times 12,50$ Metern. Das obere Stockwerk beherbergt die Kasse, Direktion, Verwaltungsräume, Cafeteria usw. sowie eine grosse Terrasse, welche herrliche Aussichten bietet.

Die Struktur ist aus Metall, und man hat dafür gesorgt, dass deren Anblick nicht monoton ist, damit die Funktionalität und moderne Gestaltung im Einklang mit der landschaftlichen und architektonischen Umgebung stehen. 